\title{
A Abordagem Clínica das Interações Pais-Bebê: Perspectivas Teóricas e Metodológicas
}

\author{
Jaqueline Wendland $^{1}$ \\ Universidade de Lille III, França
}

\begin{abstract}
Resumo
A influência das interações pais-bebê no desenvolvimento social e afetivo infantil tem sido objeto de estudo de numerosos trabalhos nas últimas três décadas. Neste artigo, examina-se, de um ponto de vista teórico e metodológico, a evolução dos estudos na área das interações pais-bebês, particularmente no campo da clínica. Aponta-se também para os temas de pesquisa que têm se revelado promissores no estudo das interações pais-bebê.

Palavras-chave: Interação; pais-bebê; desenvolvimento sócio-emocional.
\end{abstract}

The Clinical Approach of Parent-Infant Interaction: Theoretical and Methodological Perspectives

\begin{abstract}
The influence of parent-infant interaction on social and affective child development has been the object of a significant number of studies in the past three decades. This paper focuses on the evolution of the studies in the parent-infant interaction's domain. This evolution is examined from a theoretical and methodological point of view, particularly in the clinical field. Some research themes that seem to be promising in this domain are also highlighted.

Keywords: Interaction; parent-infant; socio-emotional development.
\end{abstract}

As interações pais-bebê bem como o desenvolvimento social e afetivo da criança pequena têm sido objeto de numerosos estudos nas últimas três décadas. Grande parte destes estudos teve impulso a partir do reconhecimento do potencial social inato do bebê e de seu papel ativo já nas suas primeiras interações com os pais (Brazelton, 1982). A partir de então, multiplicaramse os estudos sobre o desenvolvimento psicológico da criança no início do ciclo vital e suas interações com o mundo adulto. Estes estudos adotaram inicialmente uma perspectiva diádica (em particular a díade mãe-bebê) e mais recentemente, passaram a considerar a tríade mãepai-bebê ou o grupo familiar como um todo (FivazDepeursinge, 1998a). Paralelos aos estudos observacionais e experimentais, muitos progressos nessa área têm tido origem na colaboração cada vez mais próxima entre pesquisadores e clínicos que trabalham com a primeira infância (Lebovici, Mazet \& Rosevègue, 1990; Maury, Visier \& Montagner, 1989). Por sua vez, as implicações clínicas desses trabalhos têm sido evidenciadas na medida em que se reconhece o potencial diagnóstico, preventivo e mesmo terapêutico de avaliações da qualidade da interação pais-bebê.

Neste artigo, examinamos alguns aspectos da evolução dos estudos na área das interações pais-bebê de um ponto de visto teórico e metodológico. Desde já, cabe ressaltar

\footnotetext{
${ }^{1}$ Endereço para correspondência: Unité Petite Enfance, Groupe Hospitalier Pitié-Salpêtrière, 28, Allée Vivaldi, 75012, Paris, França. Email: JqWendland@aol.com
}

que optamos por privilegiar os estudos realizados em nossa área de atuação, ou seja, a abordagem clínica das interações.

\section{Das Concepções Psicanalíticas Pioneiras ao Estudo das Interações}

Dentre as diversas perspectivas teóricas que estudam o desenvolvimento social e afetivo do bebê, as contribuições da escola psicanalítica ocupam sem dúvida um lugar de destaque. Muito embora concepções originais de psicanalistas como Spitz (1965), Mahler (1963) e Klein (1969a, 1969b) sejam hoje em parte contestadas, as teorias psicanalíticas têm o mérito de ter insistido na importância das primeiras relações da criança enquanto experiências fundamentais no desenvolvimento do ser humano.

Uma revisão da literatura permite observar que, a despeito das discordâncias sobre a existência ou não de ego no bebê e de sua capacidade de diferenciação entre seus processos internos e o mundo externo, todos os pensadores psicanalíticos conferem especial atenção às primeiras vivências entre a mãe e o bebê. Estas seriam o protótipo, o modelo a partir do qual a criança construiria suas relações ulteriores. Além disto, alguns dos autores psicanalíticos foram pioneiros no uso da observação para fins de pesquisa clínica.

Para autores como Freud (1911/1959), Spitz (1965), Mahler (1963) e Winnicott $(1965,1987)$, ainda que o bebê apresentasse intensa atividade psíquica e que esta se construisse a partir da sua relação com o adulto, ele era 
descrito em situação bastante passiva e pouco evoluída. A concepção de Spitz do neonato como isolado do mundo exterior e incapaz de perceber estímulos sensoriais é hoje ultrapassada. Sua ignorância quanto à possibilidade de aparecimento de sorrisos face a estímulos sociais (vOz e rosto humano) muito antes da oitava semana de vida também foi criticada (Mazet \& Stoleru, 1993). Todavia, apesar dessas críticas, Spitz merece destaque por ter sido um dos primeiros psicanalistas a ter utilizado, de maneira sistemática, a observação direta de bebês, ao vivo e através de filmes. Além disso, apontou para o caráter vital da relação mãe-filho, conforme ilustram seus estudos sobre a depressão anaclítica e o hospitalismo (Spitz). Pode-se também notar que Spitz já buscava identificar as patologias da interação no que ele chamava descarrilamentos do diálogo mãe-bebê (Spitz, 1964). Da mesma forma, embora noções como autismo normal e fase simbiótica sejam hoje criticadas, os demais períodos do processo de separação-individuação descrito por Mahler permanecem atuais (Mazet \& Stoleru). Além disto, por ter descrito estes períodos a partir da observação de crianças pequenas, Mahler também pode ser considerada como uma precursora na utilização desta abordagem.

Por outro lado, contrastando com os autores mencionados acima, a concepção de Klein (1969a, 1969b) dava ao bebê uma vida psíquica bastante elaborada, pois descrevia o ego como já presente desde o nascimento. Apoiada em observações feitas durante tratamentos psicanalíticos de adultos e crianças, para Klein o bebê era capaz de sentir angústia, empregar mecanismos de defesa e estabelecer relações primitivas de objeto tanto na fantasia como na realidade. Ainda que certos aspectos das concepções de Klein pareçam hoje inverossímeis, como a complexidade da vida psíquica que ela atribui ao bebê em um período em que a maturação cerebral não parece permitir tal riqueza, suas idéias deixaram marcas profundas nos estudos sobre a relação mãe-bebê, sobretudo no campo dos distúrbios psicóticos precoces (Mazet \& Stoleru, 1993).

Dentre as concepções psicanalíticas, a teoria do apego de Bowlby (1969) é aquela que mais deu impulso às pesquisas com implicações clínicas nas últimas três décadas. Suas idéias são responsáveis por avanços significativos no estudo da relação pais-criança, pois integram às noções psicanalíticas as abordagens teóricas e metodológicas de outras escolas, tais como a etologia e a teoria dos sistemas. Técnicas como a observação direta e sistemática de comportamentos interativos tornaram-se corriqueiras no estudo da relação pais-criança e contribuíram muito para a qualidade metodológica destes estudos. Mas, sem dúvida, o destaque que têm merecido as concepções de Bowlby deve-se sobretudo às repercussões em termos de saúde mental para as quais apontaram suas idéais sobre o desenvolvimento do vínculo afetivo (Cicchetti \& Greenberg, 1991). Por outro lado, embora tenha sido concebida por um clínico e para auxiliar no diagnóstico e tratamento de distúrbios emocionais, o próprio Bowlby se disse surpreso ao constatar que sua teoria tinha dado impulso tão grande à pesquisa em psicologia do desenvolvimento, mas apenas muito lentamente influenciava o progresso da psicopatologia e da psicoterapia (Bowlby, 1988). Na medida em que os padrões de apego que a criança estabelece na sua relação com o parceiro adulto passaram a ter um valor diagnóstico, suas idéias reforçaram o intercâmbio entre a clínica e a pesquisa.

As concepções do apego de Bowlby foram bastante criticadas quando apresentadas à comunidade psicanalítica, especialmente porque elas divergiam das teorizações do pai da psicanálise. Em correspondência endereçada a Lebovici, Spitz e Anna Freud chegam a acusar Bowlby de ser um traidor da psicanálise de Freud (Lebovici, 1996). Segundo Bowlby (1969), toda criança nasce com uma necessidade social primária que, de modo freqüente, é satisfeita pelos contatos sociais com a mãe, a qual se torna usualmente sua figura de apego. Para Freud (1911/ 1959), o apego é decorrente do prazer proporcionado pela satisfação das necessidades pulsionais orais e fisiológicas. A repetição de experiências de satisfação promove o desenvolvimento do apego à figura que é ligada aos momentos de prazer, a qual é, portanto, investida libidinalmente. Contrastando com estas noções, para Bowlby, o apego decorre de predisposições biológicas não ligadas à satisfação de necessidades básicas, mas à manutenção do contato e proximidade com o parceiro adulto.

Além disto, a teoria do apego contrasta com as noções de autismo normal e período simbiótico propostas por Mahler (Mahler, Pine \& Bergman, 1975), assim como com as teorizações psicanalíticas de Winnicott $(1965,1987)$, Spitz (1965) e Freud (1911/1959), que postularam o bebê como essencialmente dependente e incapaz de discernir a realidade interna da externa. Teóricos do apego vêem o período dos primeiros meses de vida não como uma relação inicialmente simbiótica, da qual a criança emerge como diferenciada e separada, mas como uma relação que, desde seu início, permite autonomia aos parceiros no contexto de sua interação (Bretherton, 1987). Assim, a ênfase de Bowlby nas competências precoces, na tendência inata do recém-nascido para entrar em comunicação bem como na observação de seus 
comportamentos interativos influenciou profundamente os estudos sobre as interações pais-bebê.

\section{O Estudo das Interações: Algumas Considerações Metodológicas}

Pode-se notar que, retrospectivamente, os estudos do bebê e de suas interações evoluem de uma perspectiva indireta, essencialmente psicanalítica, para uma abordagem mais direta e aberta a diversos campos das ciências. $\mathrm{Na}$ primeira perspectiva, sustentada por psicanalistas de abordagem ortodoxa, trata-se de reconstruir o bebê presente em cada ser humano através das lembranças das experiências infantis e de relatos de pacientes adolescentes ou adultos submetidos à psicanálise.

Segundo Cramer (1982), neste método, o material é transmitido via linguagem e, portanto, é mediado pela dimensão simbólica de seus significados, dizendo-nos mais acerca do funcionamento psíquico infantil do que sobre os conteúdos propriamente das experiências. Se por um lado estas experiências seriam, portanto, deformadas pelas elaborações sucessivas do paciente (trata-se da história de uma história, feita e refeita diversas vezes), por outro lado, poder-se-ia argumentar que o material evocado é também enriquecido pela dimensão simbólica da linguagem e pelo contexto terapêutico e transferencial da situação analítica. Estes últimos permitem ao analista o uso de interpretações para elucidar os significados latentes dos comportamentos ou eventos relatados pelos pacientes. No entanto, a singularidade da relação analista-paciente faz com que princípios básicos da pesquisa científica, tais como o grau de concordância entre os observadores e a replicabilidade do protocolo de estudo, não possam ser atingidos. Esta corrente tem sido defendida por psicanalistas como Green, para quem a psicanálise desenvolvimentista não estuda o bebê de Freud, que só pode ser apreendido pela sua ausência e, portanto, retrospectivamente (Green, 1979).

Contrastando com esta perspectiva mais clássica, grande parte de pesquisadores e clínicos interessados no estudo do bebê têm se servido da observação direta. Nesta abordagem, os autores voltam-se especialmente para a codificação e análise dos comportamentos visíveis do bebê, definidos operacionalmente em detalhe, podendo-se obter a descrição, seqüência e freqüência dos eventos observados (Cosnier, 1984; Gottman \& Ringland, 1981). Todavia, a dificuldade de se registrar e definir operacionalmente comportamentos não observáveis diretamente, tais como a tonalidade afetiva $^{2}$ das interações

\footnotetext{
${ }^{2}$ A noção de tonalidade afetiva descreve o clima emocional ou afetivo no qual se dão as interações entre os dois parceiros (ex.: prazer, alegria, bemestar, tristeza, indiferença, aborrecimento, insegurança, distância, etc).

(Mazet, Cukier-Hemeury, Latoch, Rosemblum \& Sitbon, 1989), bem como a restrição da dinâmica do comportamento humano a um conjunto de categorias constituem algumas das limitações metodológicas desta abordagem.

Seguindo em paralelo as diferenças destas duas abordagens, o emprego de termos básicos como interação também deu origem a algumas controvérsias. Nas abordagens psicanalíticas, o termo interação ou interrelação foi, durante décadas, empregado para designar relações de objeto, ou seja, para expressar o investimento libidinal de representações mentais (objetos) pertinentes ao relacionamento entre os sujeitos (Birraux, 1995). Sob a perspectiva que estuda diretamente o bebê, a interação é definida como representando as trocas de comportamentos visíveis entre o adulto e a criança, ou seja, o foco de investigação é voltado para a descrição da interação observável (Tronick \& Cohn, 1989).

A despeito das divergências, vantagens e limitações destes métodos, pode-se dizer que, em última instância, pouca rivalidade persiste entre as perspectivas diretas e indiretas, uma vez que pesquisadores e clínicos têm tirado proveito de ambas as abordagens. Se por um lado, os clínicos passaram a admitir a necessidade do uso de uma metodologia mais rigorosa, objetiva e comparável, os pesquisadores reconheceram progressivamente a importância de, ao se estudarem as interações comportamentais, ouvir a história dos pais e das gerações que os precederam, assim como as representações associadas à criança, sua chegada ao mundo e os projetos para seu futuro. Todavia, cabe ressaltar que objetivos, linguagem e métodos distintos ainda têm dificultado o diálogo entre pesquisadores e clínicos, sobretudo no campo da pesquisa em psicoterapia (Goldfried, Borkovec, Clarkin, Johnson \& Parry, 1999; Newman \& Castonguay, 1999).

Muito embora avanços indiscutíveis tenham sido observados nesta área, uma parte considerável dos estudos ainda é suscetível de receber algumas críticas metodológicas. Entre as críticas geralmente feitas, podese salientar a questão do ambiente artificial (em geral em laboratório) e as situações de observação muito estruturadas, que podem ser consideradas como pouco representativas do contexto em que ocorrem as interações diárias entre os pais e o bebê (Belsky, 1985). Outras críticas que persistem referem-se ao número reduzido de sujeitos, aos períodos de tempo de observação, em geral curtos, ou ainda à ausência de grupos controle satisfatoriamente comparáveis aos grupos experimentais, embora estas últimas limitações derivem das dificuldades próprias às pesquisas clínicas envolvendo humanos (Lebovici, Mazet 
\& Visier, 1989; Stoleru \& Le Mer, 1995). A busca de soluções para estes problemas deu origem a uma multiplicação de metodologias de coleta e de análise de dados, freqüentemente utilizadas de maneira associada (Lebovici e cols.). Mais recentemente, os estudos nesta área têm podido contar com o auxílio de programas informáticos que permitem um ganho de tempo importante na codificação e análise estatística dos dados (ex: Interact, Dumas, 1987; The Observer, Noldus, 1996).

Como já foi mencionado, a observação ocupa um lugar central nestes estudos e atualmente ela tem sido utilizada em diversos contextos: em laboratório, em instituições como creches e hospitais, assim como no ambiente familiar. Na maioria dos casos, a observação tem por objetivo avaliar a qualidade mais ou menos saudável das interações, as competências ou as atitudes de cada participante. Todavia, mais recentemente, a observação também tem sido utilizada como instrumento terapêutico, uma vez reconhecidos os efeitos positivos da atenção e do investimento afetivo que ela pode comportar (Houzel, 1989; Jardin, 1994; Lamour \& Barraco, 1995; Lebovici, 1995).

Neste sentido, não é mais possível ignorar que a observação exerce uma influência e, em certa medida, modifica o objeto da observação. O observador, quando descreve ou filma uma díade em interação, traz consigo seus afetos, seus modos de comunicação e expressão verbal, gestual e visual, aos quais os sujeitos observados reagem, fazendo o observador reagir em contra-partida. Ambos esperam algo do outro nesta relação: o observador procura o objeto de sua pesquisa e reage face ao que observa (por exemplo privilegiando ou evitando certos ângulos de filmagem), enquanto o observado espera uma ajuda, sente-se curioso ou mesmo inconscientemente estigmatizado ao ser objeto da observação. Neste sentido, pode-se falar em fenômenos de transferência e contra-transferência entre o observador e o observado (Lebovici, 1995; Lieberman, 1998).

Uma ocasião propícia para se investigar os sentimentos de ambas as partes com relação à observação é quando os pais e o observador (em geral com formação clínica) observam juntos o filme das interações pais-bebê. Esta técnica, chamada autovideoscopia, tem mostrado sua utilidade em terapias breves pais-bebê (Field, 1980; Lebovici, 1983, 1995). Por outro lado, quando se trata de utilizar a observação em grande escala e com fins preventivos, clínicos e pesquisadores procuram estabelecer critérios mais ou menos simples e objetivos, por exemplo, para a detecção precoce de perturbações interativas ligadas ao autismo (Baron-Cohen e cols., 1996) ou à doença mental materna no pós-parto (Kumar \& Hipwell, 1996).
Examinando-se a história - bastante recente - dos trabalhos na área das interações pais-bebê, observa-se que os primeiros estudos (cf. referências citadas no próximo tópico), tanto de profissionais da clínica como da pesquisa em psicologia do desenvolvimento, muito raramente descreviam a interação do bebê com seu parceiro adulto. $\mathrm{Na}$ verdade, podemos dizer que esses estudos focalizavam o comportamento do bebê, ou do adulto que se ocupava dele, mas pouco se sabia sobre como um reagia em presença do outro. Ora, o conceito de interação pressupõe a existência de dois parceiros ou dois fenômenos que reagem reciprocamente (von Bertalanffy, 1973; Mazet e cols., 1989). Segundo Carvalho (1988), “a interação é um evento que ocorre entre indivíduos, e não nos indivíduos" (p. 514). Por outro lado, se o caráter bidirecional é portanto parte implícita no conceito de interação, durante décadas prevaleceu o modelo do parceiro adulto (em geral a mãe) todo-poderoso e organizador das interações (ao mesmo tempo ator principal, mas também único culpado em caso de perturbações). Assim, falava-se em interação (ou relação), mas estudavam-se comportamentos ou atitudes do bebê ou da mãe em determinadas situações. Todavia, ocorrendo dentro de um contexto social, estes comportamentos podem ser considerados como sociais ou interativos (Carvalho).

Atualmente, supõe-se que os estudos nesta área tenham ultrapassado a soma de fatores maternos e infantis (Mazet e cols., 1989). Muitas das teorias e modelos hoje proeminentes se inspiram na teoria transacional (Sameroff, 1975), por sua vez derivada da noção de espiral transacional introduzida por Escalona (1968). Esta teoria supõe que o ambiente e o indivíduo influenciam um ao outro num processo contínuo de desenvolvimento e de mudança. De acordo com Emde (1992), a perspectiva transacional é bastante adequada para o estudo das interações e do desenvolvimento precoce, em seus aspectos de continuidade, de adaptação e de mudança, pois pressupõe que "a individualidade não pode ser compreendida sem que sejam levados em conta os confrontos da criança aos diferentes 'ambientes"” (materno, mas também num sentido mais amplo, p.70) que ela conhecerá ao longo de sua infância. Destas noções surgiram novos conceitos na literatura, tais como sincronia (Isabella, Belsky \& von Eye, 1989), ritmicidade (Censullo, Lester \& Hoffman, 1985) e harmonização (Haft \& Slade, 1989; Stern, 1985). No entanto, dada a complexidade dos comportamentos da criança e do adulto ainda permanece a dificuldade de se conceber uma técnica de análise que capte o fenômeno interação. 
Por fim, também é importante ressaltar que os aspectos éticos das pesquisas são raramente evocados, embora a consideração dos riscos e a tomada de precauções sejam imprescindíveis, mesmo em situações aparentemente banais, mas sobretudo quando trabalhamos com populações que apresentam patologia ou risco (Lebovici, 1995). A garantia do anonimato, o direito de conhecer os resultados da pesquisa e de ser consultado quanto ao uso futuro das observações (para fins de ensino ou de divulgação científica) não podem ser negligenciados.

\section{Interações Pais-Bebê: Contextos e Temas de Pesquisa}

Pode-se também traçar algumas observações quanto à evolução dos temas e contextos dos estudos na área das interações pais-bebê. De fato, como não poderia deixar de ser, não somente a motivação pessoal do pesquisador, mas mudanças sociais bem como circunstâncias históricas direcionaram as primeiras pesquisas. O contexto de pós-guerra, a crescente necessidade de se deixar as crianças na creche e a preocupação com os efeitos negativos das separações decorrentes destas situações tiveram um papel determinante na escolha de temas e contextos de pesquisa. Assim, pode-se observar que Spitz (1965) conjugou seu interesse na psicopatologia precoce à observação do bebê em condições bem particulares: em situação de isolamento e carência, em instituições que em nada lembram o ambiente familiar. Outros precursores tais como Anna Freud (1936/1949), Burlingham (Burlingham \& Freud, 1942), David e Appell (1964), Goldfarb (1945), Robertson e mesmo Bowlby (Robertson \& Bowlby, 1952) também fizeram observações notáveis de bebês e crianças pequenas, mas muitas vezes tratava-se de observar o bebê separado de sua mãe ou em interação com um cuidador substituto. De fato, Anna Freud e Burlingham tiveram a oportunidade de observar bebês afastados de seus pais durante os bombardeios de Londres (Burlingham \& Freud). Pouco depois, a pedido da Organização Mundial da Saúde, Bowlby redigiu uma importante monografia sobre as implicações dos cuidados maternos e de sua carência para a saúde mental da criança (Bowlby, 1951).

Assim, pode-se dizer que a maior parte dos estudos clínicos nesta área tiveram como ponto de partida a questão da carência de cuidados maternos (por privação ou separação) e suas conseqüências para a criança. $\mathrm{Na}$ verdade, nesta época, mesmo antropólogos estudiosos do comportamento cultural como Mead dirigiam suas pesquisas para este tema (Mead, 1962). As repercussões destes trabalhos, embora um pouco lentas, contribuíram de maneira decisiva para mudanças nos serviços hospitalares e escolares que acolhem bebês e crianças pequenas.

Um pouco mais tardias, as contribuições da etologia para o estudo das interações pais-bebê também se tornaram numerosas e relevantes tanto em nível conceitual quanto metodológico (cf. estudos pioneiros de Lorenz (1981), Tinbergen (1951) e Harlow (Harlow \& Harlow, 1966)). Antes de mais nada, cabe lembrar que é da etologia que veio a ênfase na principal, mais simples e direta maneira de se estudar as interações: a observação. Somente através da observação minuciosa, própria à etologia, pudemos chegar à noção de competência da criança e reconhecer o papel ativo desta última na interação com o adulto ou com outras crianças (Bell \& Harper, 1977). Outro mérito da etologia é de ter salientado a importância da observação em contexto natural, ou seja, o mais próximo possível do ambiente familiar da pessoa observada. No entanto, deve-se notar que a etologia, buscando estabelecer padrões de comportamentos normais e comuns a todos os seres humanos, limita-se freqüentemente à observação de populações normais, em detrimento dos estudos psicopatológicos. Estes métodos e noções contrastam bastante com aqueles dos pioneiros psicanalistas, cujas teorizações mostravam o bebê como essencialmente passivo e dependente, e cujas observações se davam em situações muitas vezes problemáticas ou artificiais, por vezes trágicas para a criança observada.

Do ponto de vista conceitual, a etologia também forneceu conceitos tais como os de fase crítica e padrão fixo de ação, que tiveram repercussões sobretudo para a psicologia do desenvolvimento, mas também para a área clínica. Por exemplo, a noção de fase crítica está na base de alguns estudos bastante conhecidos como os de Klaus e Kennel (1982), que mostram a importância do tempo de contato mãe-recém-nascido no pós-parto para a qualidade do vínculo ulterior da mãe com a criança. Estes estudos estimularam mudanças nas práticas hospitalares, tais como a adoção do alojamento conjunto. Embora necessitem grande disponibilidade de tempo e utilizem um repertório de comportamentos extenso que torna a comparação de situações por vezes difícil, os estudos nesta área nos têm ensinado muito a respeito dos comportamentos de apego (Cyrulnik, 1989; Montagner, 1988), de imitação (Nadel, 1986) e de interação de crianças entre si (Campos de Carvalho \& Rubiano, 1996; Stamback \& Barrière, 1983). A abordagem etológica também tem se mostrado útil enquanto complemento do trabalho clínico de diagnóstico e de terapia, por exemplo, na observação das interações a domicílio de díades com dificuldades interativas (Maury e cols., 1989). 
Paralelamente aos estudos etológicos e psicanalíticos, a partir da década de 60, os trabalhos em psicologia do desenvolvimento passaram a apontar para diferenças inter-individuais presentes desde os primeiros dias de vida do bebê, tanto do ponto de vista biológico como comportamental (Wolff, 1966). Destes estudos emergiu a noção de temperamento que, por sua vez, foi fundamental para o reconhecimento da individualidade de cada bebê (Chess, 1967; Thomas, 1968). Assim, reconheciam-se em cada bebê reações, preferências e ritmos próprios. Pouco a pouco, a constatação da complexidade dos comportamentos elementares (p.ex.: sucção, olhar), da precocidade dos comportamentos perceptivos e imitativos (Fantz, 1963; Meltzoff \& Moore, 1977), e do caráter imediato do interesse pelo companheiro humano, fizeram do bebê um parceiro ativo na interação (Lewis \& Rosenblum, 1974). Investigavam-se também nesta época os comportamentos reflexos (Prechtl \& Beintema, 1968) e os estados de vigilância do bebê (Wolff). Estes últimos, como se sabe, determinam em grande parte a qualidade das respostas e reações do bebê aos estímulos e comportamentos do adulto. O bebê deixou de ser, portanto, uma criatura limitada, capaz apenas de comer, chorar e dormir e passou a ser o bebê ativo, competente e maravilhoso, que pode fazer de sua mãe a mais feliz e orgulhosa do mundo (Brazelton, 1997).

Brazelton, ao publicar e divulgar a utilização da Escala de Avaliação do Comportamento Neonatal (Brazelton, 1973) junto aos pais, deu impulso a numerosos estudos que integraram os conhecimentos sobre as competências do bebê à possibilidade de intervir e prevenir junto a díades normais ou em situação de risco (Brazelton, Nugent \& Lester, 1987; Gomes-Pedro e cols., 1987; Wendland \& Piccinini, 1998; Wendland-Carro, Piccinini \& Millar, 1999; Worobey \& Belsky, 1982). Permitir aos pais que eles descubram e admirem as competências interativas de seu bebê pode ser um passo determinante na construção da relação que eles estabelecerão com seu bebê, sobretudo em situações onde estas capacidades podem estar menos visíveis, como na prematuridade (Brazelton, 1997). Por sua vez, ao mostrarem a possibilidade de mudanças significativas na qualidade da interação, assim como na percepção dos pais sobre seus bebês, estes estudos estimularam a colaboração entre profissionais da clínica e da pesquisa da primeira infância.

Se por um lado, a descoberta do bebê competente contribuiu de maneira decisiva para a evolução dos estudos nesta área, por outro reconheceu-se cada vez mais a necessidade do ajustamento da resposta que o adulto fornece ao bebê como garantia do bom desenvolvimento emocional deste último (Bell \& Ainsworth, 1972; Belsky,
Rovine \& Taylor, 1984). Para se revelar, as competências do bebê necessitavam de um parceiro adulto atento e disponível. Assim, dois conceitos, considerados como indissociáveis, deram origem a numerosas pesquisas explorando as contribuições do adulto à interação paisbebê: a sensibilidade (sensitivity) e a responsividade (responsiveness), sobretudo da figura materna (Ainsworth, Bell \& Stayton, 1974). Estes conceitos estão intimamente associados à teoria do apego, na qual são descritos como diretamente implicados na formação dos diferentes padrões de apego no bebê (Ainsworth, Blehar, Waters \& Wall, 1978; Bretherton, 1987). Muitos estudos com intervenções e propostas de prevenção e de tratamento dos distúrbios interativos têm por objetivo a promoção destas capacidades no adulto que se ocupa com os cuidados do bebê (McDonough, 1995; Trad \& Kernberg, 1995; Whitt \& Casey, 1982). Estas noções também puderam ser mais ou menos rapidamente aproximadas de conceitos de base psicanalítica que descrevem a boa disponibilidade e o ajustamento da resposta da mãe tais como, o estado de preocupação materna primária e a noção de mãe suficientemente boa (good enough mother, Winnicott, 1969), as antecipações criativas e a empatia (Lebovici, 1983), a harmonização afetiva (affect attunement, Stern, 1985) e a disponibilidade emocional (Emde \& Sorce, 1983). Estas noções descrevem diferentes aspectos do diálogo de intenções, afetos e sinais emocionais que se estabelece entre o bebê e seu parceiro adulto desde cedo e que condiciona o desenvolvimento afetivo sadio da criança (Emde, 1992).

Vivemos hoje um momento de integração destas perspectivas e dos conhecimentos que pudemos adquirir sobre o bebê e as relações que ele estabelece com seu meio. Assim, a dicotomia entre interações observáveis e conteúdos intrapsíquicos cede pouco a pouco lugar a novas concepções, que procuram integrar tanto aspectos comportamentais quanto psicodinâmicos.

Numa perspectiva diagnóstica e terapêutica, Mazet e Stoleru (1993) descrevem diferentes perturbações ou desarmonias da qualidade das interações, que podem se expressar através do excesso ou carência de estimulações (ex.: famílias carentes, David, Lamour, Kreisler \& Harnisch, 1984; mães deprimidas, Murray, 1998), do caráter descontínuo (ex.: mães psicóticas, Lamour, 1989), não-contingente (ex.: comportamento de esquiva do olhar do outro; Greenspan \& Lieberman, 1980), paradoxal (ex.: as respostas de um membro são contraditórias à harmonia; Stern, 1977) ou regressivo das trocas diádicas (ex.: relação simbiótica persistente com relação ao processo de individuação-separação, Mahler e cols., 1975). 
Por sua vez, Lamour e Lebovici (1991) preconizam o estudo das interações em três níveis: comportamental, afetivo e fantasmático. No primeiro, observam-se as interações visuais (ex.: troca de olhares, comportamento de esquiva do contato olho-a-olho), as interações corporais (ex.: qualidade dos contatos físicos, posturas adotadas), as interações vocais ou verbais (ex.: conteúdo das verbalizações maternas, choro do bebê, contingência das respostas vocais), os comportamentos de ternura (beijos, afagos, abraços) e os sorrisos. As interações afetivas dizem respeito ao clima afetivo das interações e à influência recíproca da vida emocional do adulto e do bebê. Situações patológicas como a de uma mãe deprimida com seu bebê mostram o quanto este último é capaz de perceber e de sofrer em conseqüência dos afetos negativos, da não-contingência ou da «inexpressividade » que lhe dirige sua mãe (Field, 1987).

Por fim, a interação fantasmática se define como a influência recíproca da vida psíquica da mãe e de seu bebê. Nesta perspectiva, estuda-se a maneira como os conteúdos psíquicos de ambos os parceiros se manifestam nas interações observáveis e o modo como os fantasmas de um respondem ou modificam os fantasmas do outro (Brazelton \& Cramer, 1991; Kreisler \& Cramer, 1981). O lugar ocupado pelo bebê na problemática psíquica de sua mãe (em particular, na resolução do conflito edipiano), do casal e na história transgeracional de sua família são aspectos fundamentais nesta abordagem. Além destes aspectos, conceitos como o do bebê imaginário e bebê fantasmático, assim como o de mandato transgeracional ilustram a importância que é dada às representações neste ponto de vista (Lebovici, 1983, 1994a, 1994b). A compreensão de como se articulam representações, fantasmas, projeções, afetos, desejos e comportamentos no desenrolar das interações constitui ao mesmo tempo o desafio e o mérito do estudo das interações segundo esta abordagem. Para tanto, a formação clínica psicodinâmica do pesquisador é considerada como indispensável.

Se as contribuições da mãe foram privilegiadas em detrimento do papel do bebê durante décadas, pode-se dizer que a figura do pai também foi negligenciada até recentemente. Em 1975, Lamb designava os pais, com razão, como contribuidores esquecidos do desenvolvimento infantil. Pouco a pouco, os estudos envolvendo o pai se multiplicaram e, atualmente, o papel do pai durante a gravidez e posteriormente junto à mãe, ao bebê e à relação mãe-bebê não pode mais ser negligenciado. Enquanto instituição sociocultural, a paternidade tem se transformado rápida e constantemente ao longo da história da civilização ocidental (Knibiehler,
1988). As profundas mudanças de ordem social, cultural e familiar têm questionado a identidade do homem enquanto pai. Estas transformações encontram um paralelo na literatura psicológica e sociológica: dos novos pais ou pais modernos da década de 70, passamos a falar da "paternagem", designando o caregiving do pai (Hurstel, 1996; 1997), da paternidade de proximidade, noção psicanalítica que explora as relações entre a recente proximidade física pai-criança e a distância simbólica necessária à instalação da autoridade paterna (Cupa-Pérard e cols., 1994) e dos pais implicados nos cuidados mas diferenciados da mãe quanto a seu papel (Le Camus, 1995, 1997), para chegarmos hoje aos pais suficientemente presentes (Zaouche-Gaudron, 1997), conceito que se inspira nas noções de Winnicott a respeito da sensibilidade materna nas primeiras semanas de vida do bebê (1969).

O lugar ocupado pelo pai tem sido estudado a partir de três perspectivas: seu papel, sua função e seus comportamentos (Le Camus, 1995; Le Camus, Labrell \& Zaouche-Gaudron, 1997). Enquanto o primeiro diz respeito às condutas socialmente prescritas e publicamente anunciadas, a função está ligada aos « mecanismos de ação e efeitos da presença do pai sobre o desenvolvimento da criança » (Le Camus, p. 27). Por fim, os comportamentos paternos e a "paternagem» se referem às trocas e aos cuidados fornecidos pelo pai ao bebê e são estudados no nível das interações observáveis. A ausência ou deficiência do pai em um destes três registros parece ter, em muitos casos, repercussões negativas para a mãe e a maneira como ela vive a gravidez e a maternidade, para o desenvolvimento psíquico, social e cognitivo do bebê, assim como para a relação que a mãe estabelece com sua criança (Clément, 1993; Levy-Shiff, 1982; Mamelle, 1990; Wendland, 1995, 1999).

O crescente reconhecimento do papel do pai contribuiu sem dúvida para novos direcionamentos, sobretudo para o estudo da tríade mãe-pai-bebê e de outras interações triádicas, bem como das relações da família como um todo. A partir de uma abordagem familiar sistêmica, os trabalhos de Fivaz-Depeursinge (1998a, 1998b), embora limitando-se à microanálise comportamental, têm mostrado como o bebê é capaz de partilhar sua atenção quando se encontra na presença de seus dois pais. Por outro lado, os estudos com a família também levaram a reconsiderar as interações dos pais entre si enquanto casal e o modo como a chegada do bebê modifica o equilíbrio conjugal e conduz à assunção de novos papéis (Bydlowski, 1997; Clément, 1993; Lemaire \& Fivaz, 1989; Stoleru, 1995). 
Apesar destas mudanças, levando a perspectivas cada vez mais amplas, observamos que o contexto social em que ocorrem as interações, no sentido ecológico preconizado por Bronfenbrenner há mais de duas décadas, continua pouco considerado nestas pesquisas (Bronfenbrenner, 1977). Embora os estudos interculturais correspondam em parte a esta perspectiva, raramente a família tem sido estudada enquanto parte inserida em um sistema mais amplo (bairro, comunidade, subgrupo cultural).

\section{Perspectivas Atuais e Considerações Finais}

Os estudos atuais parecem dedicar menos interesse a questões metodológicas e divergências teóricas e focalizam sua atenção nas repercussões da qualidade das interações nos primeiros períodos da vida para o desenvolvimento ulterior do bebê. O estudo das interações entre o bebê e as pessoas que se ocupam dele em diferentes contextos e situações de vida tem implicações significativas do ponto de vista clínico. Sabe-se hoje que as perturbações destas interações constituem, em muitos casos, o único e primeiro indício de dificuldades ou perturbações em vias de instalação no bebê ou na criança pequena. Assim, o estudo das interações pais-bebê, na área da clínica, comporta uma dimensão preventiva evidente (Mazet \& Feo, 1996), que tem influenciado tanto os objetivos quanto as metodologias de pesquisa empregados.

A colaboração entre pesquisadores de diversas áreas e de profissionais que tratam o bebê tem possibilitado a realização de estudos originais, interinstitucionais e interdisciplinares, por vezes seguindo novas modalidades, tais como a de pesquisa-ação-formação (Job-Spira, Lamour, Gabel, Chambrun \& Lebovici, 1988). Neste último caso, o estudo pode comportar, além da pesquisa propriamente dita, uma ação sobre o meio ambiente, bem como a formação do pessoal engajado nos serviços que atendem pais e bebês.

Dentre os temas que têm merecido atenção crescente nesta área, podemos citar as diversas situações encontradas freqüentemente nas sociedades ocidentais atuais que expõem pais e bebê ao risco, tais como: a depressão materna (Cummings \& Davies, 1994; Mazet, Conquy, Latoch \& Rosemblum, 1990; Murray, 1998); os distúrbios mentais presentes em um dos pais (Lamour, 1989); as mães portadoras de HIV (Mandelbrot, 1998; WeilHalpern, Veber, Blanche, Duliège \& Griscelli, 1989); as mães alcoólatras ou toxicômanas (Manzano \& Palacio, 1990); os bebês que apresentam anomalias congênitas, mal-formações ou doenças crônicas (Gillot de Vries, Detraux, Vanden-Eynde, 1998); famílias migrantes ou em ruptura com seu meio de origem (Moro \& Mazet, 1998); famílias com problemas múltiplos (Greenspan e cols., 1987; Stoleru \& Morales-Huet, 1989); famílias monoparentais e mães adolescentes (Clément, 1993; Deschamps, 1993; Wendland, 1999); bebês prematuros (Harrisson \& Magill-Evans, 1996); bebês concebidos através de técnicas de reprodução assistida como a inseminação artificial (Roegiers, 1998), entre outras situações. Estes estudos têm mostrado que tanto dificuldades ligadas ao contexto (familiar, cultural, sócioeconômico) quanto a presença de problemas físicos ou mentais no bebê ou em sua família tendem a repercutir negativamente nas interações pais-bebê. Em conseqüência, podem-se observar não somente perturbações da relação ou do vínculo afetivo (ex.: carência de cuidados maternos, negligência e maus-tratos, apego inseguro, problemas do comportamento), mas também a presença de problemas psico-funcionais no bebê, que evidenciam a estreita relação entre o psíquico e o somático nas crianças pequenas (ex.: problemas do sono, perturbações digestivas e respiratórias, alergias) (Kreisler, 1995; Robert-Tissot e cols., 1989; Wendland, no prelo). Além destes aspectos, o campo das interações pais-bebê também convida o pesquisador e o clínico ao desafio de examinar as relações entre conteúdos representados (ex. representações maternas do bebê imaginário e fantasmático) e comportamentos interagidos (interação comportamental com o bebê real) (Ammaniti, 1991; Stoleru, Morales \& Grinschpoun, 1985).

Por fim, deve-se lembrar que permanecem muito atuais os estudos interculturais (Bornstein e cols., 1992, 1998; Fracasso, Lamb, Schoelmerich \& Leyendecker, 1997; Stevenson-Hinde, 1998), uma vez que aspectos sociais e particularidades culturais não somente continuam a gerar estudos comparativos, mas também têm sido levados em conta na formulação de novas abordagens terapêuticas do bebê e de sua família, tais como a etnopsiquiatria (Moro, 1993; Nathan \& Moro, 1989).

Ao concluirmos este artigo, cabe ressaltar que esta revisão não pretende ser exaustiva dos trabalhos realizados nesta área. Naturalmente, ela não pode cobrir a vasta e diversificada contribuição dos numerosos estudos desenvolvidos nas várias disciplinas que se interessam pelo bebê e suas interações. Neste sentido, deve-se também notar que ao privilegiarmos a perspectiva da clínica e do desenvolvimento sócio-emocional do bebê, por ser esta nossa área de atuação, não abordamos outros campos bastante proeminentes como o das relações entre as interações pais-bebê e o desenvolvimento cognitivo e da linguagem (Lyra, Pantoja, Cabral \& de Souza, 1995; Papousek, 1998). Assim, o estudo das primeiras relações da criança com seu meio, enquanto experiências fundamentais no desenvolvimento do ser humano, nos 
parece ser um campo fértil e relevante para a pesquisa e a prática de novas abordagens psicológicas.

\section{Referências}

Ainsworth, M.D.S., Bell, S.M. \& Stayton, D.J. (1974). Infant-mother attachment and social development: «Socialization » as a product of reciprocal responsiveness to signals. Em M.P.M. Richards (Org.), The integration of a cbild into a social world (pp. 99-135). Cambridge: University Press.

Ainsworth, M. D. S., Blehar, M.C., Waters, E. \& Wall, S. (1978) Patterns of attachment: A psychological study of the Strange Situation. Hillsdale, New Jersey: Erlbaum.

Ammaniti, M. (1991). Maternal representations during pregnancy and early infant-mother interactions. Infant Mental Health Journal, 12(3), 246-255.

Baron-Cohen, S., Cox, A., Baird, G., Sweettenham, J., Nightingale, K.M., Drew, A. \& Charman, T. (1996). Psychological markers in the detection of autism in infancy in a large population. British Journal of Psychiatry, $168,158-163$.

Bell, S. M. \& Ainsworth, M.D.S. (1972). Infant crying and maternal responsiveness. Child Development, 41, 291-311.

Bell, R. \& Harper, H. (1977). Child's effects on adults. Hillsdale, NJ: Erlbaum.

Belsky, J. (1985). Experimenting with the family in the newborn period. Child Development, 56, 407-414.

Belsky, J., Rovine, M. \& Taylor, D.G. (1984). The Pennsylvannia Infant and Family Development Project III: The origins of individual differences in infant-mother attachment: Maternal and infant contribuitions. Child Development, 55, 718-728.

Birraux, A. (1995). Prefácio. Em G. Fava-Viziello, D.N. Stern \& A. Birraux (Orgs.), Modèles psychothérapiques au premier âge (pp.19-23). Paris: Masson.

Bornstein, M. H., Haynes, O.M., Azuma, H., Galperín, C., Maital, S., Ogino, M., Painter, K., Pascual, L., Pêcheux, M-G., Rahn, C., Toda, S., Venuti, P., Vyt, A.\& Wright, B. (1998). A cross-national study of self-evaluations and attributions in parenting: Argentina, Belgium, France, Israel, Italy, Japan, and the United States. Developmental Psychology, 34, 662-676.

Bornstein, M.H., Tal, J., Rahn, C., Galperin, C.Z., Pêcheux, M.G., Lamour, M., Toda, S., Azuma, H., Ogino, M. \& Tamis-LeMonda, C.S. (1992). Functional analysis of the content of maternal speech to infants of 5 and 13 months in four cultures: Argentina, France, Japan and the United States. Developmental Psychology, 28, 593-603.

Bowlby, J. (1951). Soins maternels et santé mentale. Genève: OMS

Bowlby, J. (1969). Attachment and loss. Vol 1. New York: Basic Books.

Bowlby, J. (1988). A secure base: Parent-child attachment and healthy buman development. New York: Basic Books.

Brazelton, T. B. (1973). Neonatal Behavioral Assessment Scale. Philadelphia: Lippincott.

Brazelton, T.B. (1982). Le bébé partenaire dans l'interaction. Em T.B.

Brazelton, B. Cramer, L. Kreisler, R. Schapi \& M. Soulé, M. (Orgs.), La dynamique du nourrisson (pp. 11-27). Paris: ESF.

Brazelton, T.B. (1997). La révolution des touch points. Em M. Dugnat (Org.), Le monde relationnel du bébé (pp. 35-46). Ramonville Saint-Agne: ERES.

Brazelton, T.B. \& Cramer, B. (1991). The earliest relationship. London: Karnac

Brazelton, T.B. , Nugent, K. \& Lester, B. (1987). Neonatal Behavioral Assessment Scale. Em J. Osofsky (Org.), Handbook of infant development (pp. 780-817). New York: Wiley-Interscience.

Bretherton, I. (1987). New perspectives on attachment relations: Security, communication, and internal working models. Em J. Osofsky (Org.), Handbook of infant development (pp. 1061-1100). New York: WileyInterscience.

Bronfenbrenner, U. (1977). Toward an experimental ecology of human development. American Psychologist, 32, 513-531.

Burlingham, D. \& Freud, A. (1942). Young children in wartime: A year's work in a residential war nursery. London: Allen \& Unwin.

Bydlowski, M. (1997). La dette de vie. Paris: PUF.
Campos de Carvalho, M. I. \& Rubiano, M. R. (1996). Rede social de crianças pequenas na creche: Análise por proximidade física e atividade compartilhada. Psicologia: Teoria e Pesquisa, 12(2), 129-136.

Carvalho, A.M.A. (1988). Algumas reflexões sobre o uso da categoria interação social. Reunião Anual da Sociedade de Psicologia de Ribeirão Preto, $18,511-515$.

Censullo, M., Lester, B. \& Hoffman, J. (1985). Rhythmic patterning in mother-newborn interaction. Nursing Research, 34, 342-346.

Chess, S. (1967). The role of temperament in the child's development. Acta Paedopsychiatrica, 23, 34-51.

Cicchetti, D. \& Greenberg, M. T. (1991) The legacy of John Bowlby. Development and Psychopathology, 3, 347-350.

Clément, R. (1993) Monoparentalité et 'dysparentalité'. Em D. Fabre \& A. Savet (Orgs.), Parents au singulier. Monoparentalités: Echec on défi? (pp. 126132) Paris: Editions Autrement.

Cosnier, J. (1984). Observation directe des interactions précoces ou les bases de l'épigenèse interactionnelle. Psychiatrie de l'enfant, 27(1), 107126.

Cramer, B. (1982). La psychiatrie du bébé: Une introduction. Em T.B. Brazelton, B. Cramer, L. Kreisler, R. Schapi, \& M. Soulé (Orgs.), La dynamique du nourrrisson (pp. 5-12) Paris: ESF.

Cummings, E. M. \& Davies, P.T. (1994). Maternal depression and child development. Journal of Child Psychology and Psychiatry, 35 (1), 73-112.

Cupa-Pérard, D., Moinet, I., Chassin, F., Thoret, N., Bel, C. \& Valdès, L. (1994). Devenir père ou la grossesse du père. Revue de Médecine Psychosomatique, 37/38, 85-106.

Cyrulnik, B. (1989). Sous le signe du lien. Paris: Hachette.

David, M. \& Appell, G. (1964). Etude des facteurs de carence affective dans une pouponnière. Psychiatrie de l'enfant, 4(2), 401-442.

David, M., Lamour, M., Kreisler, A. \& Harnisch, R. (1984). Recherche sur les nourrissons de familles carencées. Psycbiatrie de l'enfant, 27(1), 175222.

Deschamps, J-P. (1993). Mères adolescentes, parents adolescentes. Em D. Favre \& A. Savet (Orgs.), Parents an singulier. Monoparentalités: Echec on défi? (pp. 190-203) Paris: Editions Autrement.

Dumas, J. E. (1987). Interact- A computer-based coding and data management system to assess family interactions. Em R.J. Prinz (Org.), Advances in behavioral assessment of children and families (pp.177-202). New York: JAI Press.

Emde, R.N. (1992). Génétique des émotions (développement terminé et interminable). Em Ph.Mazet \& S. Lebovici (Orgs.), Emotions et affects chez le bébé et ses partenaires (pp. 59- 132). Paris: ESHEL.

Emde, R. \& Sorce, J.E. (1983). The rewards of infancy: Emotional availability and maternal referencing. Em J.D. Call, E. Galenson, \& N.R. Tyson (Orgs.), Frontiers of infant psychiatry (pp. 17-30). New York: Basic Books.

Escalona, S. (1968). The roots of individuality. Chicago: Adline.

Fantz, R. L. (1963). Patterns of vision in newborn infants. Science, 140, 296297.

Field, T.M. (1980). Interactions of preterm and term infants with their lower- and middle-class teenage and adult mothers. Em T. M. Field (Org.), High risk infants and children (pp. 113-132). New York: Academic Press.

Field, T. M. (1987). Affective and interactives disturbances in infants. Em J.D. Osofsky (Org.), Handbook of infant development (pp. 972-1005). New York: Wiley \& Sons.

Fivaz-Depeursinge, E. (1998a). Infant's triangulation strategies: A new issue in development. The Signal, 6(3-4), 11-16.

Fivaz-Depeursinge, E. (1998b). Au commencement était l'action. La microanalyse: Pourquoi et comment? Bulletin du Groupe WAIMH-Francophone, 5(2), 5-9.

Fracasso, M. P., Lamb, M. E., Schoelmerich, A. \& Leyendecker, B. (1997). The ecology of mother-infant interaction in Euro-American and immigrant Central American families living in the United States. International Journal of Behavioral Development, 20(2), 207-217.

Freud, A. (1949). Le moi et les mécanismes de défense. Paris: PUF. (Original publicado em 1936) 
Freud, S. (1959). Os dois princípios do suceder psíquico. Em J. Salomão (Org.) Obras completas de Sigmund Freud (Vol. 7, pp.321-329). Rio de Janeiro: Delta. (Original publicado em 1911)

Gillot de Vries, F., Detraux, J-J. \& Vanden-Eynde, S. (1998). Approche du vécu maternel suite à l'annonce d'une anomalie foetale. Em P. Mazet \& S. Lebovici (Orgs.), Psychiatrie périnatale. Parents et bébés: Du projet d'enfant aux premiers mois de vie (pp. 157-167) Paris: PUF.

Goldfarb, W. (1945). Effects of psychological deprivation in infancy and subsequent stimulation. American Journal of Psychiatry, 102, 18-33.

Goldfried, M. R., Borkovec, T. D., Clarkin, J. F., Johnson, L. D. \& Parry, G. (1999). Toward the development of a clinically useful approach to psychotherapy research. Journal of Clinical Psychology, 55, 1385-1405.

Gomes-Pedro, J., Monteiro, M.B., Carvalho,A. Patrício, M.F., Garcia, F.T. \& Barbosa, A. (1987). Early intervention and mother-infant interaction during the first three months of life [Resumo]. Em The International Society for the Study for Behavioral Development (Org.). Resumos ISSBD (p.67). Tóquio: Wiley.

Gottman, J. M. \& Ringland, J. T. (1981). The analysis of dominance and bidirectionality in social development. Child Development, 52, 393-412.

Green, A. (1979). L'enfant modèle. Nouvelle Revue de Psychanalyse, 19, 27-48.

Greenspan, S. \& Lieberman, A. (1980). Infants, mothers and their interactions. A quantitative approach to developmental assessment. Em S.I. Greenspan \& G.H Pollock (Orgs.), The course of life (Vol. 1 pp. 271-310). Washington: Government Printing Office.

Greenspan, S., Wieder, S., Nover, R., Lieberman, A., Lourie, R. \& Robinson, M. (1987). Infants in multirisk families. Madison: International Universities.

Haft, W.L. \& Slade, A. (1989). Affect attunement and maternal attachment. Infant Mental Health Journal, 10(3), 157-172.

Harlow, H. F. \& Harlow, M. K. (1966). Learning to love. American Scientist, $54,244-272$.

Harrisson, M. J. \& Magill-Evans, J. (1996). Mother and father interactions over the first year with term and preterm infants. Research in Nursing and Health, 19(6), 451-459.

Houzel, D. (1989). Penser les bébés. Réflexions sur l’observation des nourrissons. Revue de Médecine Psychosomatique, 19, 27-38.

Hurstel, F. (1996). Rôle social et fonctions psychologiques du père._Informations Sociales, 56, 8-17.

Hurstel, F. (1997). Identité masculine, inversion des rôles parentaux, fonction paternelle. Enfance, 3, 411-423.

Isabella, R.A., Belsky, J. \& von Eye, A .(1989). Origins of infant-mother attachment: An examination of interactional synchrony during the infant's first year. Developmental Psychology, 25, 12-21.

Jardin, F. (1994). Une stratégie de soins précoces du bébé et de la parentalité. Apport de l'observation analytique du bébé en crèche à des fins thérapeutiques. Devenir, 6(2), 87-103.

Job-Spira, N., Lamour, M., Gabel, M., Chambrun, J. de, \& Lebovici, S. (1988). Recherche-action sur la prévention de la maltraitance chez le très jeune enfant: Méthodologie et premiers résultats. Archives Françaises de Pédiatrie, 45(4), 277-285.

Klaus, M. \& Kennel, J. (1982). Parent-infant bonding. St.Louis: C.V. Mosby.

Klein, M. (1969a). Algumas conclusões teóricas sobre a vida emocional do bebê. Em M. Klein, P. Heimann, S. Isaacs \& J. Riviere (Orgs.), Os progressos da psicanálise (pp. 216-249). Rio de Janeiro: Zahar. (Original publicado em 1952)

Klein, M. (1969b). Sobre a observação do comportamento dos bebês. Em M. Klein, P. Heimann, S. Isaacs \& J. Riviere (Orgs.), Os progressos da psicanálise (pp. 256-289). Rio de Janeiro: Zahar. (Original publicado em 1952)

Knibiehler, Y. (1988, novembro). Le rôle des pères à travers l'Histoire. Revue Francaise des Affaires Sociales, 27-39.

Kreisler, L. (1995). La perspective psychosomatique dans la psychopathologie du nourrisson. Em G. Fava-Viziello, D. Stern, D. \& A. Birraux (Orgs.), Modèles psychothérapiques au premier âge. De la théorie à l'intervention (pp. 159-200). Paris: Masson.

Kreisler, L. \& Cramer, B. (1981). Sur les bases cliniques de la psychiatrie du nourrisson. Psychiatrie de l'enfant, 24(1), 223-263.
Kumar, R. \& Hipwell, A.E. (1996). Development of a clinical rating scale to assess mother-infant interaction in a psychiatric mother and baby unit. British Journal of Psycbiatry, 169, 18-26.

Lamb, M.E. (1975). Fathers: Forgotten contributors to child development. Human Development, 18, 245-266.

Lamour, M. (1989). Les nourrissons de parents psychotiques. Em S. Lebovici \& F. Weil-Halpern (Orgs.), Psychopathologie du bébé (pp. 655-673). Paris: PUF.

Lamour, M. \& Barraco, M. (1995, abril-junho). Dans les situations à haut risque. L'observation des nourrissons comme mode d'abord thérapeutique. Dialogue, 81-92.

Lamour, M. \& Lebovici, S. (1991). Les interactions du nourrisson avec ses partenaires: Evaluation et modes d'abord préventives et thérapeutiques. Psychiatrie de l'enfant, 34(1), 171-275.

Le Camus, J. (1995). Pères et bébés. Paris: L'Harmattan.

Le Camus, J. (1997). Présentation du numéro. Enfance, 3, 325-336.

Le Camus, J. , Labrell, F. \& Zaouche-Gaudron, C (1997). Le rôle du père dans le développement de l'enfant. Paris: Nathan.

Lebovici, S. (1983). Le nourrisson, la mère et le psychanalyste. Paris: Le Centurion.

Lebovici, S. (1994a). Les interactions fantasmatiques. Revue de Médecine Psychosomatique, 37/38, 39-50.

Lebovici, S. (1994b). L'homme dans le bébé. Revue Française de Psychanalyse, 3, $661-680$.

Lebovici, S. (1995). Techniques de l'observation du très jeune enfant. Em S. Lebovici, R. Diatkine \& M. Soulé (Orgs.), Nouveau traité de psychiatrie de l'enfant et de l'adolescent (pp. 549-561). Paris: PUF.

Lebovici, S. (1996). John Bowlby décrit à travers quelques souvenirs personnels. Bulletin WAIMH- France, 3(2), 4-5.

Lebovici, S., Mazet, P. \& Rosevègue, P. (1990). Des pédiatres et des psychiatres collaborent à une recherche psychopathologique sur les interactions très précoces en vue d'une action préventive. Devenir, 1, 70-75.

Lebovici, S., Mazet, P. \& Visier, J.P. (1989). Avant-propos. Em S. Lebovici, P. Mazet \& J-P Visier (Orgs.), L'évaluation des interactions précoces entre le bébé et ses partenaires (pp. 9-12). Paris: ESHEL.

Lemaire, J-G \& Fivaz, E. (1989) L'organisation familiale et la psychopathologie du bébé. Em S. Lebovici \& F. Weil-Halpern (Orgs.), Psychopathologie du Bébé (pp.89-106). Paris: PUF.

Levy-Shiff, R. (1982). The effects of father absence on young children in mother-headed families. Child Development, 53, 1400-1405.

Lewis, M. \& Rosemblum, L. A. (1974). The effect of the infant on its caregiver. New York: John Willey.

Lieberman, A. (1998). A perspective on infant mental health. The Signal, 6 (1), 11-12.

Lorenz, K. (1981). The foundations of etology. New York: Springer-Verlag.

Lyra, M. C., Pantoja, A. P., Cabral, E. A. \& de Souza, M. (1995). A produção vocal do bebê: construção partilhada pela díade. Psicologia: Teoria e Pesquisa, 11(1), 1-6.

Mahler, M. (1963). Thoughts about development and individuation. The Psychoanalytic Study of the Child, 18, 307-324.

Mahler, M., Pine, F. \& Bergman, A. (1975). The psychological birth of the buman infant. New York: Basic Books.

Mamelle, N. (1990). Composantes psychologiques dans l'étiologie de la prématurité. Gynécologie et psychosomatique, 3, 5-10.

Mandelbrot, L. (1998). Séropositivité au VIH et grossesse. Em P. Mazet \& S. Lebovici (Orgs.), Psychiatrie périnatale. Parents et bébés: Du projet d'enfant aux premiers mois de vie (pp. 187-197). Paris: PUF.

Manzano, J. \& Palacio, F. (1990). Les enfants de parents toxicomanes: une étude clinique. Neuropsychiatrie de l'enfance, 38(6), 378-384.

Maury, M., Visier, J-P, \& Montagner, H. (1989). Intérêt d'une approche intégrée, psychiatrique et éthologique des interactions entre le bébé et sa mère et de leurs dysfonctionnements. Em S. Lebovici, P. Mazet \& J-P Visier (Orgs.), L'évaluation des interactions précoces entre le bébé et ses partenaires (pp. 71-97). Paris: ESHEL.

Mazet, P., Conquy, L., Latoch, J. \& Rosemblum, O. (1990). Bebés et mères déprimées. Devenir, 4, 71-80. 
Mazet, P., Cukier-Hemeury, F., Latoch, J., Rosenblum, O. \& Sitbon, H. (1989). Etude historique et critique. Em S. Lebovici, P. Mazet \& J-P Visier (Orgs.), L'évaluation des interactions précoces entre le bébé et ses partenaires (pp. 15-39). Paris: ESHEL.

Mazet, P. \& Feo, A. (1996). Interactions précoces e recherche. Pour la recherche, 9, 2-6.

Mazet, P. \& Stoleru, S. (1993). Psychopathologie du nourrisson et du jeune enfant. Paris: Masson.

McDonough, S. (1995). L'aide à l'interaction: Une technique pour le traitement des troubles relationnels précoces. Em G. Fava-Viziello, D.N. Stern \& A. Birraux (Orgs.), Modèles psychothérapiques au premier âge: De la théorie à lintervention (pp. 225-237). Paris: Masson.

Mead, M. (1962). La carence de soins maternels du point de vue de l'anthropologie culturelle. OMS, 14, 44-62.

Meltzoff, A. \& Moore, M.K. (1977). Imitation of facial and manual gestures by human neonates. Science, 198, 75-78.

Montagner, H. (1988). L'attachement, les débuts de la tendresse. Paris: Odile Jacob.

Moro, M. R. (1993). Coconstruire l'interaction parents-enfants: Du sens culturel au sens individuel. Em A. Yahyaoui (Org.), Destins de femmes, réalités de l'exil. (pp. 199-216) Grenoble: La pensée sauvage.

Moro, M.R. \& Mazet, P. (1998). Parents et enfants «sans larmes ». Nécessité d'une approche psychologique et culturelle de la périnatalité. Em P. Mazet \& S. Lebovici (Orgs.), Psychiatrie périnatale. Parents et bébés: Du projet d'enfant aux premiers mois de vie. (pp. 489-504) Paris: PUF.

Murray, L. (1998). L'impact de la dépression du post-partum sur le développement de l'enfant. Em P. Mazet \& S. Lebovici (Orgs.), Psychiatrie périnatale. Parents et bébés: Du projet d'enfant aux premiers mois de vie (pp. 287298). Paris: PUF.

Nadel, J. (1986). Imitation et communication entre jeunes enfants. Paris: PUF.

Nathan, T. \& Moro, M.R. (1989). Enfants de «djinné »: Evaluation ethnopsychanalytique des interactions précoces. Em S. Lebovici, P. Mazet \& J-P Visier (Orgs.), L'évaluation des interactions précoces entre le bébé et ses partenaires (pp. 307-339). Paris: ESHEL.

Newman, M.G. \& Castonguay, L.G. (1999). Reflecting on current challenges and future directions in psychotherapy: What can be learned from dialogues between clinicians, researchers, and policy makers? Journal of Clinical Psychology, 55, 1407-1413.

Noldus Technology Inc. (1996). The observer. Professional system for collection, analysis and management of observational data. Documento comercial publicitário. Wageningen (Holanda).

Papousek, M. (1998). Regulatory functions of preverbal communication: Compensatory support and failures. [Resumos] Em The International Society for the Study for Behavioral Development (Org.), Resumos ISSBD (p. 317). Basel: H. Leidenfrost AG.

Prechtl, H.F.R. \& Beintema, J. (1968). The neurological examination of the fullterm newborn infant. Clinics in developmental medicine $\left(\mathrm{n}^{\circ} 28\right)$. London: Spastic International Medical Publications \& Heinemann Medical.

Robertson, J. \& Bowlby, J. (1952). Responses of young children to separation from their mother. Courrier du Centre International de l'Enfance, 2, 131-142.

Robert-Tissot, C., Rusconi-Serpa, S., Bachman, J-P., Besson, G., Cramer, B., Knauer, D., de Muralt, M., Palacio, F. \& Stern, D. N. (1989). Le questionnaire symptom check-list: Evaluation des troubles psychofonctionnels de la petite enfance. Em S. Lebovici, P. Mazet \& J-P Visier (Orgs.), L'Evaluation des interactions précoces entre le bébé et ses partenaires (pp. 179-215). Paris: ESHEL.

Roegiers, L. (1998). Aide médicale à la procréation et facteurs de risque. Entre psychiatrie et éthique. Em P. Mazet \& S. Lebovici (Orgs.), Psychiatrie périnatale. Parents et bébés: Du projet d'enfant aux premiers mois de vie (pp. 81-90). Paris: PUF.

Sameroff, A. J. (1975). Early influences on development: Fact or fancy? Merrill-Palmer Quarterly, 21, 267-294.

Spitz, R. (1964). The derailment of dialogue: Stimulus overload, action cycles and the completion gradient. Journal of the American Psychoanalytic Association, 12, 752-775.

Spitz, R. (1965). The first year of life. New York: International Universities Press.
Stambak, M. \& Barrière, M. (1983). Les bébés entre eux. Paris: PUF. Stern, D.N. (1977). Mère, enfant. Les premières relations. Bruxelas: Pierre Mardaga Stern, D.N. (1985). La conversation d'avant le langage. Autrement, 72, 118121.

Stevenson-Hinde, J. (1998). Parenting in different cultures: Time to focus. Developmental Psychology, 34, 698-700.

Stoleru, S. (1995). Le couple et le projet d'enfant. L'étape initiale du passage à la parentalité. Neuropsychiatrie de l'enfance, 43(4-5), 164-170.

Stoleru, S. \& Le Mer, M.N. (1995). Problèmes de méthodologie de la recherche. Présentation de l'entretien semi-structuré multi-registres. Devenir, 7(3), 55-75.

Stoleru, S., Morales, M. \& Grinschpoun, M.-F. (1985). De l'enfant fantasmatique de la grossesse à l'interaction mère-nourrisson. Psychiatrie de l'Enfant, 28(2), 441-484.

Stoleru, S. \& Morales-Huet, M. (1989). Psychothérapies mère-nourrisson dans les familles à problèmes multiples. Paris: PUF.

Thomas, A.S. (1968). Temperament and behavior disorders in children. New York: New York University Press.

Tinbergen, N. (1951). The study of instinct. Oxford: Oxford University Press.

Trad, P. V. \& Kernberg, P. F. (1995). L'anticipation du changement évolutif à court terme et ses implications dans la relation mère-enfant. Em G. Fava-Viziello, D.N. Stern \& A. Birraux (Orgs.), Modèles psychothérapiques au premier âge: De la théorie à lintervention (pp. 239-264). Paris: Masson.

Tronick, E.Z. \& Cohn, J.F. (1989). Infant-mother face-to-face interaction: Age and gender differences in coordination and the occurrence of miscoordination. Child Development, 60, 85-92.

Von Bertalanffy, L. (1973). Théorie générale des systèmes. Paris: Dunod.

Weil-Halpern, F., Veber, F., Blanche, S., Duliège, A.M. \& Griscelli, C. (1989). Conséquences de l'infection par le virus HIV de la mère sur les interactions mère-bébé. Em S. Lebovici, P. Mazet \& J-P Visier (Orgs.) L'Evaluation des interactions précoces entre le bébé et ses partenaires (pp. 295-304). Paris: ESHEL.

Wendland, J. (1995). Les pièges du «être heureux à deux »: Transmission intergénérationnelle et risques de la monoparentalité. Dissertação não-publicada. Diploma Universitário de Psicopatologia do Bebê (Especialização). Universidade Paris XIII, Paris, França.

Wendland, J. (1999). Devenir mère en centre maternel: Une étude clinique longitudinale et comparative auprès de jeunes mères célibataires. Tese de Doutorado em Psicologia Clínica não-publicada. Universidade Paris XIII, Paris, França.

Wendland, J. (no prelo). Problemas psico-funcionais do bebê: Resultados de uma investigação junto à jovens mães solteiras. Em M. LeitgelGilles (Org.), Hospitalização do bebê: Um enfoque multidisciplinar. Salvador: Agalma.

Wendland, J. \& Piccinini, C. (1998). Effets d'une intervention précoce visant à favoriser la qualité de l'interaction mère-bébé. Psychiatrie de l'enfant, 61(1), 187-217.

Wendland-Carro, J., Piccinini, C. \& Millar, S. (1999). The role of an early intervention on enhancing the quality of mother-infant interaction in low income families. Child Development, 70, 713-721.

Winnicott, D.W. (1965). The maturational processes and the facilitating environment. London: Hogarth.

Winnicott, D. W. (1969). La préoccupation maternelle primaire. Em D. Winnicott (Org.), De la pédiatrie à la psychanalyse (pp. 168-174). Paris: Payot. (Original publicado em 1956)

Winnicott, D. W. (1987). Babies and their mothers. Reading, MA: AddisonWesley.

Whitt, J.K. \& Casey, P.H. (1982). The mother-infant relationship and infant development: The effect of pediatric intervention. Child Development, 53, 948-956.

Wolff, P.H. (1966). The causes, control and organisation of behaviour in the neonate. Psychological Issues, 5, 7-11.

Worobey, J. \& Belsky, J. (1982). Employing the Brazelton scale to influence mothering: An experimental comparison of three strategies. Developmental Psychology, 18, 736-743. 
Zaouche-Gaudron, C. (1997). La différenciation paternelle et le père

Recebido em 01.08.2000 suffisament présent. Neuropsychiatrie de l'Enfance et de l'Adolescence, 45(3),

Revisado em 05.11.2000 $153-161$.

Aceito em 05.12.2000

Sobre a autora:

\section{Jaqueline Wendland}

Psicóloga, Mestre em Psicologia do Desenvolvimento (Universidade Federal do Rio Grande do Sul, Porto Alegre, Brasil) e Doutora em Psicologia Clínica (Universidade de Paris XIII, França). Professora de Psicologia Clínica e Psicopatologia na Universidade de Lille III (França) e psicóloga clínica na Unité Petite Enfance, Hospital Pitié-Salpêtrière, Paris (França). 